Pacific Journal of Mathematic 


\section{ON A CHARACTERIZATION USING RANDOM SUMS}

J. R. Choike, I. I. Kotlarski and V. M. Smith

Let $X_{1}, X_{2}$, and $X_{3}$ be independent random variables and let $Z_{1}=X_{1}+X_{3}$ and $Z_{2}=X_{2}+X_{3}$. It is known that if the characteristic functions of $X_{k}, k=1,2,3$, do not vanish then the distribution of $\left(Z_{1}, Z_{2}\right)$ determines the distributions of $X_{1}$, $X_{2}$, and $X_{3}$ up to a shift. The aim of this paper is to prove a result of a similar nature using sums of a random number of random variables. We shall use $\sim$ for "has the same distribution as," r. v. for "random variable," ch. f. for "characteristic function," and p.g.f. for "probability generating function."

TheOREM 1. Let $N, X_{1}, X_{2}, \cdots, Y_{1}, Y_{2}, \cdots$ be independent r.v.'s where $X_{n} \sim X, Y_{n} \sim Y, n=1,2, \cdots$, and $X$ and $Y$ are nondegenerate real-valued r.v.'s having ch.f.'s $\varphi$ and $\psi$, respectively, which are of bounded variation on every finite interval. Let $N$ be a nonnegative integer-valued r.v. with p.g.f.

$$
Q(s)=p_{0}+\sum_{n=1}^{\infty} p_{n} s^{n}, \quad|s| \leqq 1, \quad p_{n}=P(N=n)
$$

and $0<E N=m<\infty$. Assume that there is a neighborhood of 1 relative to the unit disk such that $Q^{-1}$ exists in this neighborhood. Denote

$$
\begin{aligned}
& U=0 \text { for } N=0, U=X_{1}+X_{2}+\cdots+X_{N} \text { for } N>0, \text { and } \\
& V=0 \text { for } N=0, V=Y_{1}+Y_{2}+\cdots+Y_{N} \text { for } N>0 .
\end{aligned}
$$

Then the distribution of $(U, V)$ uniquely determines the distribution of $N$.

Proof. Since $N, X_{1}, X_{2}, \cdots, Y_{1}, Y_{2}, \cdots$ are independent r.v.'s, the ch.f. of $(U, V), \varphi_{(U, V)}$, satisfies the following:

$$
\begin{aligned}
& \mathcal{P}_{\langle r, V\rangle}(r, t)=E\left(e^{i r C^{+}+i t r}\right) \\
& =E\left(e^{i r l+i t\ulcorner} \mid N=0\right) \cdot P(N=0)+\sum_{n=1}^{\infty} E\left(e^{i r l+i t \Gamma^{\top}} \mid N=n\right) \cdot P(N=n) \\
& =E(1) \cdot p_{0}+\sum_{n=1}^{\infty} E\left(e^{i r\left(X_{1}+\cdots+X_{n}\right)+i t\left(Y_{1}+\cdots+Y_{n}\right)}\right) \cdot p_{n} \\
& =p_{0}+\sum_{n=1}^{\infty}\left[E\left(e^{i r . X}\right) \cdot E\left(e^{i t r}\right)\right]^{n} \cdot p_{n} \\
& =p_{0}+\sum_{n=1}^{\infty}\left[\varphi\left(r^{\prime}\right) \cdot \psi(t)\right]^{n} \cdot p_{n} \\
& =Q(\varphi(r) \cdot \psi(t)), \quad r, t \in R \text {. }
\end{aligned}
$$


Suppose there are other r.v.'s $N^{*}, X_{1}^{*}, X_{2}^{*}, \cdots, Y_{1}^{*}, Y_{2}^{*}, \cdots$, satisfying the assumptions. By repeating the above procedure denoting $U^{*}$ and $V^{*}$ similarly we obtain

$$
\varphi_{\left(U^{*}, V^{*}\right)}(r, t)=Q^{*}\left(\varphi^{*}(r) \cdot \psi^{*}(t)\right), \quad r, t \in R .
$$

Since $\left(U^{*}, V^{*}\right)$ has the same distribution as $(U, V)$, their ch.f.'s are identical; thus,

$$
Q^{*}\left(\phi^{*}(r) \cdot \psi^{*}(t)\right)=Q(\varphi(r) \cdot \psi(t)), \quad r, t \in R .
$$

Relation (2) is a functional equation and from this equation it will be shown that $Q^{*}=Q$.

The function $Q$ is analytic inside the disk, thus the image of a domain under $Q$ is a domain. There is a neighborhood of 1 relative to the unit disk such that $Q^{*-1}$ exists and is analytic in this neighborhood. Thus there exists a neighborhood $A$ of 1 relative to the unit disk such that $Q^{*-1}$ exists and is analytic in $Q(A)$. Define

$$
q(s)=Q^{*-1}(Q(s)) \quad s \in A .
$$

Note that $q$ is analytic in $A$ and maps $A$ into the unit disk. It can be assumed without loss of generality that $0 \notin A$.

Using relations (2) and (3),

$$
q(\varphi(r) \cdot \psi(t))=\varphi^{*}(r) \cdot \psi^{*}(t) \quad r, t \in R, \varphi(r) \cdot \psi(t) \in A .
$$

By alternately allowing $r=0$ and $t=0$ it is found that $q\left(\varphi\left(r^{\circ}\right)\right)=$ $\phi^{*}(r)$ and $q(\psi(t))=\psi^{*}(t)$. Substituting these into relation (4)

$$
q(\varphi(r) \cdot \psi(t))=q(\varphi(r)) \cdot q(\psi(t)) \quad r, t \in R, \varphi(r) \cdot \psi(t) \in A .
$$

Since $0 \notin A$, there exist continuous functions $\varphi_{0}$ and $\psi_{0}$ such that $\varphi(r)=e^{\varphi_{0}(r)}$ and $\psi(t)=e^{\psi_{0}(t)}$ and $\varphi_{0}(0)=\psi_{0}(0)=0$ where $\varphi(r) \cdot \psi(t) \in A$. Since $\varphi$ and $\psi$ are of bounded variation on finite intervals, $\varphi_{0}$ and $\psi_{0}$ are of bounded variation on finite intervals. Define

$$
q_{0}(b)=\ln q\left(e^{b}\right) \quad e^{b} \in A,
$$

where we take the branch for which $\ln 1=0$. Then from relation (6)

$$
\begin{aligned}
q_{0}\left(\varphi_{0}(r)+\psi_{0}(t)\right) & =\ln q\left(e^{\varphi_{0}(r)+\psi_{0}(t)}\right) \\
& =\ln q(\varphi(r) \cdot \psi(t)) \\
& =\ln [q(\varphi(r)) \cdot q(\psi(t))] \\
& =\ln q(\varphi(r))+\ln q(\psi(t)) \\
& =\ln q\left(e^{\varphi_{0}(r)}\right)+\ln q\left(e^{\psi_{0}(t)}\right) \\
& =q_{0}\left(\varphi_{0}(r)\right)+q_{0}\left(\psi_{0}(t)\right), \quad \varphi(r) \cdot \psi(t) \in A .
\end{aligned}
$$


Consider the following integrals obtained by using equation (7)

$$
\begin{aligned}
\int_{0}^{\beta} q_{0}\left(\varphi_{0}(\alpha)+\psi_{0}(t)\right) d \psi_{0}(t) & =\int_{0}^{\beta}\left[q_{0}\left(\varphi_{0}(\alpha)\right)+q_{0}\left(\psi_{0}(t)\right)\right] d \psi_{0}(t) \\
& =q_{0}\left(\varphi_{0}(\alpha)\right) \cdot \psi_{0}(\beta)+\int_{0}^{\beta} q_{0}\left(\psi_{0}(t)\right) d \psi_{0}(t)
\end{aligned}
$$

and

$$
\begin{aligned}
\int_{0}^{\alpha} q_{0}\left(\varphi_{0}(r)+\psi_{0}(\beta)\right) d \varphi_{0}(r) & =\int_{0}^{\alpha}\left[q_{0}\left(\varphi_{0}(r)\right)+q_{0}\left(\psi_{0}(\beta)\right)\right] d \varphi_{0}(r) \\
& =\int_{0}^{\alpha} q_{0}\left(\varphi_{0}(r)\right) d \varphi_{0}(r)+q_{0}\left(\psi_{0}(\beta)\right) \cdot \varphi_{0}(\alpha)
\end{aligned}
$$

where $\alpha$ and $\beta$ are fixed real numbers such that $\varphi(r) \cdot \psi(t) \in A$ for $0 \leqq r \leqq \alpha$ and $0 \leqq t \leqq \beta$. These integrals exist because $\varphi_{0}$ and $\psi_{0}$ are of bounded variation on finite intervals and $q_{0}$ is analytic. Using a change of variables on relations (8) and (9), the following integrals are obtained.

$$
\begin{aligned}
& \int_{\psi_{0}(\alpha)}^{\varphi_{0}(\alpha)+\psi_{0}(\beta)} q_{0}(v) d v=q_{0}\left(\varphi_{0}(\alpha)\right) \cdot \psi_{0}(\beta)+\int_{0}^{\psi_{0}(\beta)} q_{0}(v) d v . \\
& \int_{\psi_{0}(\beta)}^{\varphi_{0}(\alpha)+\psi_{0}(\beta)} q_{0}(v) d v=q_{0}\left(\psi_{0}(\beta)\right) \cdot \varphi_{0}(\alpha)+\int_{0}^{\varphi_{0}(\alpha)} q_{0}(v) d v .
\end{aligned}
$$

By adding equations (10) and (11) right sides to left sides the following equation is obtained,

$$
\begin{aligned}
& \int_{0}^{\varphi_{0}(\alpha)+\psi_{0}(\beta)} q_{0}(v) d v+q_{0}\left(\psi_{0}(\beta)\right) \cdot \varphi_{0}(\alpha) \\
& =\int_{0}^{\varphi_{0}(\alpha)+\gamma_{0}(\beta)} q_{0}(v) d v+q_{0}\left(\varphi_{0}(\alpha)\right) \cdot \psi_{0}(\beta) .
\end{aligned}
$$

From this it is seen that

$$
q_{0}\left(\psi_{0}(\beta)\right) \cdot \varphi_{0}(\alpha)=q_{0}\left(\varphi_{0}(\alpha)\right) \cdot \psi_{0}(\beta) .
$$

Since $X$ and $Y$ are nondegenerate, $|\varphi(r)|<1$ and $|\psi(t)|<1$ almost everywhere. Thus $\varphi_{0}(\alpha)$ and $\psi_{0}(\beta)$ are different from zero almost everywhere and

$$
\frac{q_{0}\left(\psi_{0}(\beta)\right)}{\psi_{0}(\beta)}=\frac{q_{0}\left(\varphi_{0}(\alpha)\right)}{\varphi_{0}(\alpha)}
$$

Since the choice of $\alpha$ is independent of $\beta$

$$
q_{0}\left(\varphi_{0}(\alpha)\right)=c \varphi_{0}(\alpha) \quad \text { where } c \text { is a complex number . }
$$

Since $q_{0}(b)=\ln q\left(e^{b}\right), q(s)=s^{c}$ for $s \in A$.

Since $c$ is complex, $c=a+i b$ where $a, b \in R$. Thus $Q^{*-1}(Q(s))=$ 
$s^{a+i b}$ for $s \in A$ since $q(s)=Q^{*-1}(Q(s))$. Since $A$ is a relative neighborhood of 1 , there is a segment of the real line $[\delta, 1] \subset A$ where $0<$ $\delta<1$. The function $Q$ maps the unit disk into the unit disk, and $Q^{*-1}$ maps $Q(A)$ into the unit disk. For $s \in[\delta, 1], s^{c}=e^{c \ln s}=e^{a \ln s+i b \ln s}=$ $e^{a \ln s} \cdot e^{i b \ln s}$. Since $\left|s^{c}\right| \leqq 1, a \ln s \leqq 0$ for $s \in[\delta, 1]$. Thus $a \geqq 0$ since $\ln s \leqq 0$. The function $Q(s)$ is real for $s$ a real number and $Q^{*-1}(Q(s))$ is real for $Q(s)$ a real number. Thus for $s \in[\delta, 1], s^{c}$ is a real number and $b \ln s=0 \bmod (2 \pi)$. Thus $b=0$ and $c=a \geqq 0$.

Since $Q^{*-1}(Q(s))=s^{c}$ for $s \in A$, then $Q(s)=Q^{*}\left(s^{c}\right)$ for $s \in A$. The functions $Q, Q^{*}, s^{c}$ are analytic for $0<|s|<1$, thus $Q(s)=Q^{*}\left(s^{c}\right)$ for $0<|s|<1$. Suppose $c=0$. Then $Q(s)=Q^{*}(1)=1$ for $0<|s|<1$. This implies that $E N=0$ which is a contradiction. Thus $c \neq 0$. Since the expectation of $N$ and $N^{*}$ exist $\lim _{s \rightarrow 1} Q^{\prime}(s)=\lim _{s \rightarrow 1} c s^{c-1} Q^{* \prime}\left(s^{c}\right)$ or $m=c m$. Thus $c=1$ and $Q(s)=Q^{*}(s)$ for all $|s| \leqq 1$.

REMARK. A characterization for the distribution of $N$ has been found using the assumptions of Theorem 1 . The following shows that the assumption "that there is a neighborhood of 1 relative to the unit disk such that $Q^{-1}$ exists in this neighborhood" is redundant.

THEOREM 2. Let $N$ be a nonnegative integer-valued r.v. with p.g.f.

$$
Q(s)=p_{0}+\sum_{n=1}^{\infty} p_{n} s^{n}, \quad|s| \leqq 1, \quad p_{n}=P(N=n) .
$$

If $0<E N<+\infty$, then $Q$ is one-to-one in a relative neighborhood of 1 .

Proof. Let $D=\{s:|s|<1, s \in C\}$ and $Q(s)=u(s)+i v(s)$ where $u$ and $v$ are real-valued functions.

Let

$$
G\left(x_{1}, y_{1}, x_{2}, y_{2}\right)=\left(\begin{array}{l}
u_{x}\left(x_{1}, y_{1}\right), u_{y}\left(x_{1}, y_{1}\right) \\
v_{x}\left(x_{2}, y_{2}\right), v_{y}\left(x_{2}, y_{2}\right)
\end{array}\right)
$$

where $x_{1}+i y_{1}, x_{2}+i y_{2}$ are in $\bar{D}$. The function $Q$ is analytic in $D$ if and only if $u$ and $v$ are differentiable in $D$ and satisfy the Cauchy-Riemann equations.

Let

$$
f(x, y)=\left(\begin{array}{l}
u(x, y) \\
v(x, y)
\end{array}\right) \quad x+i y \in C .
$$

Thus $f(x, y)$ is differentiable in $D, f^{\prime}$ may be represented by the Jacobian matrix of $f$, 


$$
f^{\prime}(x, y)=\left(\begin{array}{l}
u_{x}(x, y), u_{y}(x, y) \\
v_{x}(x, y), v_{y}(x, y)
\end{array}\right) \quad x+i y \in C,
$$

and $f^{\prime}(x, y)$ has continuous existension to $\bar{D}$.

The mapping $\operatorname{det}\left[G\left(x_{1}, y_{1}, x_{2}, y_{2}\right)\right]: R^{4} \rightarrow R$ is continuous on $\bar{D} \times$ $\bar{D} \subset R^{4}$. But

$$
\operatorname{det}\left[G\left(x_{1}, y_{1}, x_{2}, y_{2}\right)\right]=u_{x}\left(x_{1}, y_{1}\right) \cdot v_{y}\left(x_{2}, y_{2}\right)-u_{y}\left(x_{1}, y_{1}\right) \cdot v_{x}\left(x_{2}, y_{2}\right) \text {. }
$$

Since $\operatorname{det}[G(1,0,1,0)]=\left|Q^{\prime}(1)\right|^{2} \neq 0$, there exists a convex neighborhood of 1 such that $\operatorname{det}\left[G\left(x_{1}, y_{1}, x_{2}, y_{2}\right] \neq 0\right.$ is this convex (closed) neighborhood. Without loss of generality, we assume $\operatorname{det}\left[G\left(x_{1}, y_{1}\right.\right.$, $\left.\left.x_{2}, y_{2}\right)\right] \neq 0$ for all $x_{1}+i y_{1}, x_{2}+i y_{2} \in \bar{D}$.

Let $\vec{c}, \vec{d} \in \bar{D}$. By the Mean Value Theorem for vector-valued functions

$$
f(\vec{c})-f(\vec{d})=G\left(\overrightarrow{c_{1}}, \overrightarrow{c_{2}}\right)(\vec{c}-\vec{d})
$$

where $\vec{c}_{j}=\left(1-t_{j}\right) \vec{c}+t_{j} \vec{d}, j=1,2$, for some $t_{j} \in(0,1)$. Note that $\vec{c}_{j} \in D, j=1,2$.

Since $\operatorname{det} G\left[\left(x_{1}, y_{1}, x_{2}, y_{2}\right)\right] \neq 0$, the matrix $G\left(x_{1}, y_{1}, x_{2}, y_{2}\right)$ represents a one-to-one linear map. Thus, if $\vec{c} \neq \vec{d}$, then $f(\vec{c}) \neq f(\vec{d})$. Thus, $Q$ is one-to-one in a relative neighborhood of 1 .

Note that in Theorem 1 nothing is said about the distributions of $X$ and $Y$. The following example will show that more assumptions are needed in order to determine the distributions of $X$ and $Y$.

EXAMPLE 1. Let $N$ and $N^{*}$ be distributed according to the p.g.f. $Q(s)=s^{2},|s| \leqq 1$. Let $X$ be distributed according to the characteristic function $\varphi(r)=1-2|r| \mid \pi$ for $|r| \leqq \pi$ and $\varphi(r)$ is periodic with period $2 \pi$, and let $X^{*} \sim|\varphi(r)|$. Let $Y \sim Y^{*} \sim \psi(t)$ where $\psi(t)$ is any nonvanishing real-valued ch.f. $(U, V) \sim\left(U^{*}, V^{*}\right)$ since

$Q^{*}\left(\varphi^{*}(r) \cdot \psi^{*}(t)\right)=Q(\varphi(r) \cdot \psi(t)), \quad r, t \in R \quad$ although $\quad \varphi^{*}\left(r^{*}\right) \neq \varphi\left(r^{*}\right)$.

Thus more conditions must be imposed in order to prove Theorem 3.

THeOREM 3. Let $N, X_{1}, X_{2}, \cdots, Y_{1}, Y_{2}, \cdots$ be r.v.'s satisfying the assumptions of Theorem 1, and $U$ and $V$ be defined as in Theorem 1. Then the distribution of $(U, V)$ uniquely determines the distributions of $X$ and $Y$ if one of the following conditions holds: 
(i) The characteristic functions $\varphi$ and $\psi$ are analytic at zero.

(ii) There is a relative neighborhood $B$ of 1 such that $\varphi(r)$. $\psi(t) \in B, r, t \in R$, and $Q$ is one-to-one on $B$.

Proof. From the proof of Theorem $1 Q^{*}=Q$ and

$$
Q\left(\varphi^{*}(r) \cdot \psi^{*}(t)\right)=Q(\varphi(r) \cdot \psi(t)) \quad r, t \in R .
$$

Thus by alternately letting $r=0$ and $t=0$

$$
Q\left(\varphi^{*}(r)\right)=Q(\varphi(r)) \text { and } Q\left(\psi^{*}(t)\right)=Q(\psi(t)) \quad r, t \in R .
$$

If condition (ii) is assumed, then it is clear that $\varphi^{*}=\varphi$ and $\psi^{*}=\psi$.

If conition (i) is assumed, then as before, $Q$ has a local inverse at one and $\varphi^{*}(\boldsymbol{r})=\varphi(r)$ and $\psi^{*}(t)=\psi(t)$ for $r, t$ in some neighborhood of zero. But since the functions are analytic ch.f.'s, $\varphi^{*}=\varphi$ and $\psi^{*}=\psi$.

Thus the distributions of $X$ and $Y$ are determined uniquely.

The following theorem has a proof very similar to that of Theorem 1.

Theorem 4. Let $N, X_{1}, X_{2}, \cdots, Y_{1}, Y_{2}, \cdots$ be independent r.v.'s with $X_{n} \sim X, Y_{n} \sim Y, n=1,2, \cdots$, where $X$ and $Y$ are symmetric real-valued nondegenerate r.v.'s having ch.f.'s $\phi$ and $\psi$, respectively, with $0 \leqq \varphi(r) \leqq 1$ and $0 \leqq \psi(t) \leqq 1, r, t \in R$. Let $N$ be a nonnegative integer-valued r.v. with p.g.f.

$$
Q(s)=p_{0}+\sum_{n=1}^{\infty} p_{n} s^{n}, \quad|s| \leqq 1, \quad p_{n}=P(N=n)
$$

where $0<E N=m<\infty$.

Denote $U$ and $V$ as in Theorem 1.

Then the distribution of $(U, V)$ uniquely determines the distributions of $X, Y$, and $N$.

Proof. The proof of this theorem is the same as the proof of Theorem 1 up to relation (2). At this point the fact that $\varphi$ and $\psi$ are nonnegative real-valued functions can be used to simplify the proof. Since $E N>0$ and $E N^{*}>0, Q$ and $Q^{*}$ are strictly increasing on the interval $[0,1]$. Thus the inverse of $Q$ and $Q^{*}$ exist as functions from $\left[p_{0}, 1\right]$ and $\left[p_{0}^{*}, 1\right]$, respectively, onto $[0,1]$. Without loss of generality $p_{0}^{*} \leqq p_{0}$. By letting

$$
q(s)=Q^{*-1}(Q(s)) \quad s \in[0,1]
$$


and using relation (2) in Theorem 1

$$
q(\varphi(r) \cdot \psi(t))=\varphi^{*}(r) \cdot \psi^{*}(t) \quad r, t \in R .
$$

Note that $q$ is continuous since $Q^{*}$ and $Q$ are continuous. Taking alternately $r=0$ and $t=0$ and substituting in equation (2) gives

$$
q(\varphi(r) \cdot \psi(t))=q(\varphi(r)) \cdot q(\psi(t)) \quad r, t \in R .
$$

Denote $A=\{a: a=\varphi(r), r \in R\}$ and $B=\{b: b=\psi(t), t \in R\}$. Since $X$ and $Y$ are nondegenerate, $\varphi$ and $\psi$ are not identically equal to 1 . Since $\varphi$ and $\psi$ are real-valued, continuous, and $\varphi(0)=\psi(0)=1$, there is an interval $[c, 1], 0<c<1$, such that $[c, 1] \subset A \cap B$. Thus

$$
q(a b)=q(a) \cdot q(b) \quad \text { for } \quad a, b, a b \in[c, 1] .
$$

From [1], $q(s)=s^{k}$ for $s \in[c, 1]$ and $k$ some real number. Using the same argument as in Theorem $1, k=1$ and $Q^{*}(s)=Q(s),|s| \leqq 1$. Thus the distribution of $N$ is uniquely determined.

Using relation (1), $q(s)=s$, and relation (2) yields $\varphi^{*}(r)=\varphi(r)$, $r \in R$, and $\psi^{*}(t)=\psi^{\prime}(t), t \in R$. Thus the distributions of $X$ and $Y$ are uniquely determined.

REMARKs. In each of the theorems we have assumed $0<E N=$ $m<+\infty$. This assumption can be replaced by the assumption, "There exists a fixed smallest positive index $j_{0}$, such that $p_{j_{0}}>0$." The theorems can be generalized if $X$ and $Y$ are random variables taking values in a locally compact Abelian group or taking values in a locally convex topological vector space if appropriate assumptions are made.

\section{REFERENCES}

1. J. Aczél, Lectures on Functional Equations and Their Applications, New York: Academic Press, 1966.

2. C. Burrill, Measure, Integration, and Probability, New York: McGraw-Hill Book Company, 1972.

3. I. Kotlarski, On characterizing the gamma and the normal distribution, Pacific J. Math., 20 (1967), 69-76.

4. E. Lukacs, Characteristic Functions, 2nd ed. London: Charles Griffin and Company Limited, 1970.

5. R. Nevanlinna and V. Paatero, Introduction to Complex Analysis, London: AddisonWesley Publishing Company, 1969.

6. H. Sagan, Advanced Calculus, Boston: Houghton Miffin Company, 1974.

Received December 20, 1976 and in revised form May 30, 1980.

Oklahoma State University

Stillwater, OK 74074

AND

The Aerospace Corp.

El Segundo, CA 90245 



\section{PACIFIC JOURNAL OF MATHEMATICS}

\section{EDITORS}

DoNALD BABBITT (Managing Editor)

University of Galifornia

Los Angeles, California 90024

HUgo RossI

University of Utah

Salt Lake City, UT 84112

C. C. MOORE AND ANDREW OGG

University of California

Berkeley, CA 94720
J. DugunduI

Department of Mathematics

University of Southern California

Los Angeles, California 90007

R. Finn and J. Milgram

Stanford University

Stanford, California 94305

\section{ASSOCIATE EDITORS}
E. F. BeCKenbaCh
B. H. NeumanN
F. WOLF
K. YoSHIDA

\section{SUPPORTING INSTITUTIONS}

UNIVERSITY OF BRITISH COLUMBIA

CALIFORNIA INSTITUTE OF TECHNOLOGY

UNIVERSITY OF CALIFORNIA

MONTANA STATE UNIVERSITY

UNIVERSITY OF NEVADA, RENO

NEW MEXICO STATE UNIVERSITY

OREGON STATE UNIVERSITY

UNIVERSITY OF OREGON
UNIVERSITY OF SOUTHERN CALIFONIA STANFORD UNIVERSITY UNIVERSITY OF HAWAII UNIVERSITY OF TOKYO UNIVERSITY OF UTAH WASHINGTON STATE UNIVERSITY UNIVERSITY OF WASHINGTON 


\section{Pacific Journal of Mathematics}

\section{Vol. 91, No. $1 \quad$ November, 1980}

Harvey Leslie Abbott, Extremal problems on nonaveraging and nondividing

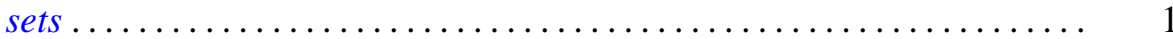

Marine Bruce Abrahamse and Stephen D. Fisher, Mapping intervals to

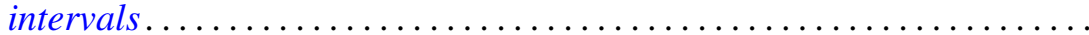

William Wells Adams, The best two-dimensional Diophantine

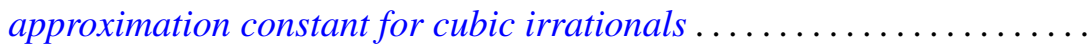

Marilyn Breen, A quantitative version of Krasnosel'skiu 's theorem in

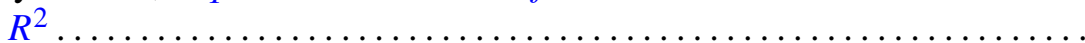

Stephen LaVern Campbell, Linear operators for which $T^{*} T$ and $T T^{*}$

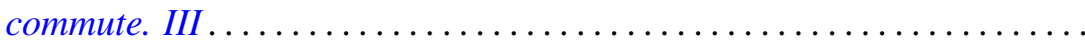

Zvonko Cerin, On cellular decompositions of Hilbert cube manifolds ......

J. R. Choike, Ignacy I. Kotlarski and V. M. Smith, On a characterization

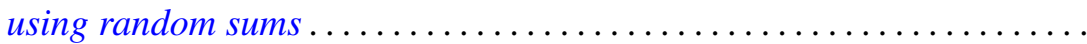

Karl-Theodor Eisele, Direct factorizations of measures .............. 79

Douglas Harris, Every space is a path component space ............. 95

John P. Holmes and Arthur Argyle Sagle, Analytic H-spaces, Campbell-Hausdorff formula, and alternative algebras.............

Richard Howard Hudson and Kenneth S. Williams, Some new residuacity criteria ..........................................

V. Karunakaran and Michael Robert Ziegler, The radius of starlikeness for a class of regular functions defined by an integral ....

Ka-Sing Lau, On the Banach spaces of functions with bounded upper

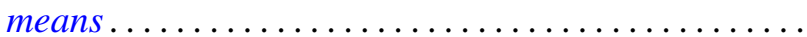

Daniel Paul Maki, On determining regular behavior from the recurrence formula for orthogonal polynomials................

Stephen Joseph McAdam, Asymptotic prime divisors and going down...

Douglas Edward Miller, Borel selectors for separated quotients ..

Kent Morrison, The scheme of finite-dimensional representations of an algebra

Donald P. Story, A characterization of the local Radon-Nikodým property by tensor products

Arne Stray, Two applications of the Schur-Nevanlinna algorithm ...

N. B. Tinberg, The Levi decomposition of a split $(B, N)$-pair ...

Charles Irvin Vinsonhaler and William Jennings Wickless, A theorem on quasi-pure-projective torsion free abelian groups of finite rank... 